\title{
On Branching Numbers of Normal Manifolds*
}

\author{
Daniel Ralph \\ TR 92-1283 \\ (replaces TR 91-1255) \\ May 1992
}

Department of Computer Science Cornell University

Ithaca, NY 14853-7501

\footnotetext{
"This research was supported in part by the U.S. Army Research Office through the Mathematical Sciences Institute, Cornell University and in part by the National Science Foundation, the Air Force Office of Scientific Research and the Office of naval research, under grant DMS-8920550.
} 



\title{
On Branching Numbers of Normal Manifolds ${ }^{1}$
}

\author{
Daniel Ralph \\ Cornell University \\ Department of Computer Science, Upson Hall \\ Ithaca, NY 14853
}

November 1991. Revised February 1992.

\footnotetext{
${ }^{1}$ This research was supported in part by the U.S. Army Research Office through the Mathematical Sciences Institute, Cornell University, and in part by the National Science Foundation, the Air Force Office of Scientific Research, and the Office of Naval Research, under grant DMS-8920550.
} 



\begin{abstract}
Let $\mathcal{M}$ be a finite piecewise linear (pl) manifold of $\mathbb{R}^{n}$, and $P: \mathbb{R}^{n} \rightarrow \mathbb{R}^{n}$ be pl with respect to $\mathcal{M}$, i.e. $P$ is affine on each set in $\mathcal{M}$. The branching number of $\mathcal{M}, \mathrm{Kuhn}$ and Löwen [8], is the maximum number of sets in $\mathcal{M}$ that can contain any common face of codimension 2. [8, Thm. 5.3] shows that if $\mathcal{M}$ has branching number less than or equal to 4 , then $P$ is a homeomorphism if and only if it is coherently oriented, i.e. the determinants of $P$ on the sets in $\mathcal{M}$ have the same nonzero sign.

Let $C$ be a nonempty polyhedral convex set in $\mathbb{R}^{n}$, and $A \in \mathbb{R}^{n \times n}$. Robinson [14] defines a finite pl manifold $\mathcal{N}_{C}$ of $\mathbb{R}^{n}$ called the normal manifold of $C$; and the normal map $A_{C}: \mathbb{R}^{n} \rightarrow \mathbb{R}^{n}$ induced by $(A, C)$, which is pl with respect to $\mathcal{N}_{C}$. [14, Thm. 4.3] shows that $A_{C}$ is homeomorphic if and only if it is coherently oriented.

We show that $\mathcal{N}_{C}$ has branching number less than or equal to 4 , hence Robinson's result is actually a corollary of Kuhn and Löwen's.
\end{abstract}

\title{
Key Words
}

Piecewise linear, piecewise affine, branching number, normal map, pl-normal, normal manifold, homeomorphism, coherently oriented

\section{Abbreviated Title}

On Branching Numbers of $\mathcal{N}_{C}$. 



\section{Introduction}

Kuhn and Löwen [8] and Robinson [14] have provided deep and elegant results that characterize the homeomorphic members of certain classes of piecewise linear (pl) mappings. The purpose of this paper is to show that the class of mappings examined by Robinson is contained in the class of mappings examined by Kuhn and Löwen.

Let $\mathcal{M}$ be a finite piecewise linear ( $\mathrm{pl}$ ) manifold of $\mathbb{R}^{n}$, and $P: \mathbb{R}^{n} \rightarrow \mathbb{R}^{n}$ be pl with respect to $\mathcal{M}$, i.e. $P$ is affine on each set in $\mathcal{M}$. The branching number of $\mathcal{M}$, Kuhn and Löwen [8], is the maximum number of sets in $\mathcal{M}$ that can contain any common face of codimension 2. [8, Thm. 5.3] shows that if $\mathcal{M}$ has branching number less than or equal to 4 , then $P$ is a homeomorphism if and only if it is coherently oriented, i.e. the determinants of $P$ on each set in $\mathcal{M}$ have the same nonzero sign.

Let $C$ be a nonempty polyhedral convex set in real $n$-dimensional space, $\mathbb{R}^{n}$, and $A$ belong to the space of real $n \times n$ matrices, $\mathbb{R}^{n \times n}$. Robinson [14] defines a finite pl manifold $\mathcal{N}_{C}$ of $\mathbb{R}^{n}$ [14, Prop. 2.4] called the normal manifold of $C$; and the normal map $A_{C}: \mathbb{R}^{n} \rightarrow \mathbb{R}^{n}$ induced by $(A, C)$, which is pl with respect to $\mathcal{N}_{C}$ [14, Prop. 2.5]. The main result [14, Thm. 4.3] shows that $A_{C}$ is homeomorphic if and only if it is coherently oriented; see Ralph [10] for an alternative proof, and Robinson [15] for a much simpler proof in the special case that $A$ is symmetric. It is pointed out in $[14, \S 1]$ that if $\mathcal{N}_{C}$ could be shown to have branching number less than or equal to 4 , then $[14, \mathrm{Thm} .4 .3]$ would follow from [8, Thm. 5.3]. Indeed, showing that normal manifolds have this branching number property is precisely our goal. 
Throughout the paper, $C$ and $A$ will be as above. Let $\pi_{C}$ be the Euclidean projection onto $C$, that is $\pi_{C}$ maps each point of $\mathbb{R}^{n}$ to its nearest point in $C$ with respect to the Euclidean norm. The identity in $\mathbb{R}^{n \times n}$ is $I$.

Definition 1 [14] The normal mapping induced by $(A, C)$ is the mapping from $\mathbb{R}^{n}$ to $\mathbb{R}^{n}$ given by

$$
A_{C} \stackrel{\text { def }}{=} A \pi_{C}+I-\pi_{C}
$$

Such normal mappings are called pl-normal mappings.

Pl-normal systems are important in many optimization and equilibrium problems. They arise directly from variational inequalities or, equivalently, generalized equations specified by linear mappings and polyhedral convex sets; and indirectly as approximations to such systems specified by smooth nonlinear functions over polyhedral convex sets. For example, see Robinson [16], Ralph [11]. Perhaps the best known of these problems is the linear complementarity problem $[3,9]$ which can be posed as a pl-normal equation $A_{C}(x)=a$, for appropriate $A \in \mathbb{R}^{n \times n}$ and $a \in \mathbb{R}^{n}$, by taking $C$ to be the nonnegative orthant $\mathbb{R}_{+}^{n}$.

The question of necessary and sufficient conditions for $\mathrm{pl}$ mappings to be homeomorphic was answered for the linear complementarity problem by Samelson, Thrall and Wesler [18], and later by Murty [9]. Other work on the general question includes Fujisawa and Kuh [6], Rheinboldt and Vandergraft [12], Kojima and Saigal [7], Schramm [19], and Eaves and Rothblum [5].

The remainder of this section will be spent giving rigor to our introductory remarks on $\mathrm{pl}$ manifolds, branching numbers, coherent orientation, and 
homeomorphism results. $§ 2$ contains the main result on branching numbers, Proposition 12.

We recall the following terms.

- A set in $\mathbb{R}^{n}$ is polyhedral convex if it is the intersection of finitely many closed half-spaces.

- A face of a convex set $D \subset \mathbb{R}^{n}$ is a subset $F$ of $D$ such that for $x_{1}, x_{2} \in D$ and $0<t<1$, if $t x_{1}+(1-t) x_{2}$ belongs to $F$ then $x_{1}, x_{2}$ belong to $F$.

\section{Definition 2}

1. An $n$-cell [4], or chamber [8], (in $\mathbb{R}^{n}$ ) is a polyhedral convex set with nonempty interior in $\mathbb{R}^{n}$.

2. A pl manifold, or chamber system [8], of $\mathbb{R}^{n}$ is a locally finite family of $n$-cells covering $\mathbb{R}^{n}$, any two of which intersect in a (possibly empty) common face. A pl manifold of $\mathbb{R}^{n}$ is finite if it consists of finitely many $n$-cells.

3. Let $\mathcal{M}$ be a pl manifold of $\mathbb{R}^{n}$. The branching number [8] of $\mathcal{M}$ is the least $b \in\{1,2, \ldots, \infty\}$ such that every face of an $n$-cell of $\mathcal{M}$ which has codimension 2 is contained in at most $b$-cells.

We note that, according to the proof of [14, Prop. 2.4], any pl manifold $\mathcal{M}$

of $\mathbb{R}^{n}$ is also a subdivided pl manifold of dimension $n$ in the sense of Eaves [4]; namely, in addition to satisfying the requirements of Definition 2.2 , it has the property that each face of dimension $n-1$ of an $n$-cell of $\mathcal{M}$ is contained in at most two $n$-cells of $\mathcal{M}$. 
Definition 3 Let $P: \mathbb{R}^{n} \rightarrow \mathbb{R}^{n}$, and $\mathcal{M}$ be a pl manifold of $\mathbb{R}^{n}$.

1. $P$ is $\mathrm{pl}$ with respect to $\mathcal{M}$ if for each $\sigma \in \mathcal{M}$, there are $A_{\sigma} \in \mathbb{R}^{n \times n}$ and $b_{\sigma} \in \mathbb{R}^{n}$ such that

$$
P(x)=A_{\sigma} x+b_{\sigma}, \quad \forall x \in \sigma .
$$

2. If $P$ pl with respect to $\mathcal{M}$ and the matrices $A_{\sigma}(\sigma \in \mathcal{M})$ are as above, the determinants of $P$ are the determinants of the matrices $A_{\sigma}$.

Suppose $P, \mathcal{M}$ and $A_{\sigma}(\sigma \in \mathcal{M})$ are as in the definition. Note that $\mathcal{M}$ is not uniquely determined by $P$. The set of matrices $\left\{A_{\sigma} \mid \sigma \in \mathcal{M}\right\}$ and the set of their determinants are, however, uniquely determined by $P$ and are independent of $\mathcal{M}$. Furthermore it is easy to see, since $\mathcal{M}$ is locally finite, that $P$ is not only continuous but locally Lipschitz; and that $P$ is a Lipschitz mapping if $\mathcal{M}$ is finite.

We now present the characterization theorems of Kuhn and Löwen [8], and Robinson [14], respectively.

Theorem 4 Suppose $\mathcal{M}$ is a finite pl manifold of $\mathbb{R}^{n}$ with branching number less than or equal to 4. If $P: \mathbb{R}^{n} \rightarrow \mathbb{R}^{n}$ is pl with respect to $\mathcal{M}$, then it is a homeomorphism if and only if it is coherently oriented.

Proof Let $P$ be pl with respect to $\mathcal{M}$. [8, Thm. 5.3] says that if $P$ is proper, i.e. $P^{-1}(D)$ is compact for any compact set $D \subset \mathbb{R}^{n}$, then it is bijective if and only if it is coherently oriented.

Suppose $P$ is bijective. It is easy to see that $P(\mathcal{M}) \stackrel{\text { def }}{=}\{P(\sigma) \mid \sigma \in \mathcal{M}\}$ is a pl manifold of $\mathbb{R}^{n}$, and that $P^{-1}$ is pl with respect to $P(\mathcal{M})$. Hence both 
$P$ and $P^{-1}$ are continuous, and $P$ is a homeomorphism. To conclude, we note that each of the conditions of bijectivity and coherent orientation imply that $P$ is invertible on each of its $n$-cells, from which it follows, by finiteness of $\mathcal{M}$, that $P$ is proper.

Theorem $5 A_{C}$ is a homeomorphism if and only if $A_{C}$ is coherently oriented.

Proof According to [14, Thm. 4.3], $A_{C}$ is a Lipschitzian homeomorphism if and only if $A_{C}$ is coherently oriented. Now $A_{C}$ is pl with respect to the finite pl manifold $\mathcal{N}_{C}[14, \mathrm{Thm} .2 .5(\mathrm{i})]$ hence, as noted previously, $A_{C}$ is Lipschitz, and the stated result follows.

Details of $\mathcal{N}_{C}$ will be given in $\S 2$. It follows from our main result, Proposition 12 , that the branching number of $\mathcal{N}_{C}$ is less than or equal to 4 , hence we can apply Theorem 4 directly to the pl-normal mapping $A_{C}$ to obtain Theorem 5 .

\section{Normal manifolds, their quotients and lo- cal structure}

Further notation:

- The relative interior of a set $F$ in $\mathbb{R}^{n}$ is denoted ri $F$. A basic fact $\left[17\right.$, Thm. 6.1] is that each nonempty convex set in $\mathbb{R}^{n}$ has nonempty relative interior.

- The affine hull of a set $F \subset \mathbb{R}^{n}$ is denoted aff $F$. The linear subspace parallel to aff $F$ is denoted par $F$. The linear subpace parallel to the largest affine subspace of $F$ is denoted lin $F$. 
- A set $K$ in $\mathbb{R}^{n}$ is a cone if for each $\alpha>0$ and $k \in K, \alpha k \in K$. If $K$ is nonempty cone, the polar cone of $K$ is

$$
K^{\circ} \stackrel{\text { def }}{=}\left\{x \in \mathbb{R}^{n} \mid\langle x, k\rangle \leq 0, \forall k \in K\right\}
$$

The polar cone of the empty set is defined to be the empty set.

- The normal cone to $C$ at $\hat{c} \in C$ is

$$
N_{C}(\hat{c}) \stackrel{\text { def }}{=}\left\{\nu \in \mathbb{R}^{n} \mid\langle\nu, c-\hat{c}\rangle \leq 0, \forall c \in C\right\}
$$

$N_{C}(\hat{c})$ is defined to be the empty set if $\hat{c} \notin C$.

The tangent cone to $C$ at $\hat{c} \in \mathbb{R}^{n}$, is $T_{C}(\hat{c}) \stackrel{\text { def }}{=} N_{C}(\hat{c})^{\circ}$.

- If $X$ is a nonempty set in $\mathbb{R}^{n}, X^{\perp}$ is the set of vectors $z \in \mathbb{R}^{n}$ such that $\langle z, x\rangle=0$ for each $x \in X$.

- The critical cone [13] to $C$ at $x \in \mathbb{R}^{n}$ is $T_{C}\left(\pi_{C}(x)\right) \cap\left[x-\pi_{C}(x)\right]^{\perp}$.

By convention, the sum or cartesian product of two sets in $\mathbb{R}^{n}$, one of which is empty, is also empty.

Proposition 6 [14, Prop. 2.1, 2.4, 2.5]

1. For each face $F$ of $C$ and each point $f$ in the relative interior of $F$, the normal cone to $C$ at $f$ is the same (nonempty) set. Denote this by $N_{F}$. Also, for any point $c \in F$,

$$
N_{F}=N_{C}(c) \cap(F-c)^{\perp} .
$$


2. The family of sets $F+N_{F}$, where $F$ is any nonempty face of $C$, is a pl manifold of $\mathbb{R}^{n}$.

3. On each $n$-cell $F+N_{F}$, where $F$ is a nonempty face of $C, A_{C}$ acts as an affine mapping:

$$
A_{C}(f+\nu)=A f+\nu, \quad \forall f \in F, \nu \in N_{F} .
$$

The constancy of $N_{C}(x)$ for $x$ in the relative interior of a face $F$ of $C$ is also shown by Burke and Moré [2, Thm. 2.3].

\section{Definition 7}

1. Let $F$ be a nonempty face of $C$ and $f \in \operatorname{ri} F$. The normal cone of $C$ on $F\left(\right.$ in $\left.\mathbb{R}^{n}\right)$ is $N_{F} \stackrel{\text { def }}{=} N_{C}(f)$.

2. The normal manifold [14] of $C$ (in $\mathbb{R}^{n}$ ), denoted $\mathcal{N}_{C}$, is the family of sets $F+N_{F}$ where $F$ is a nonempty face of $C$.

Since $C$ is polyhedral convex, it has finitely many nonempty faces $F$ (see [17, Thm. 19.1]). So part 2 of Proposition 6 says that $\mathcal{N}_{C}$ is a finite pl manifold of $\mathbb{R}^{n}$. Part 3 says that $A_{C}$ is pl with respect to $\mathcal{N}_{C}$.

Our first task is to show one-to-one correspondence between the nonempty faces of $C$ and $n$-cells of $\mathcal{N}_{C}$.

\section{Proposition 8}

1. Let $F$ be a nonempty face of $C$.

The mapping $x \mapsto\left(\pi_{C}(x), x-\pi_{C}(x)\right)$, where $x \in F+N_{F}$, is a bijection from $F+N_{F}$ to $F \times N_{F}$. 
2. The mapping $F \mapsto F+N_{F}$ is a bijection from the family of nonempty faces $F$ of $C$ to $\mathcal{N}_{C}$.

\section{Proof}

1. Let $\mu(x) \stackrel{\text { def }}{=}\left(\pi_{C}(x), x-\pi_{C}(x)\right)$. Clearly the addition mapping + is the inverse of $\mu$. Also $+: F \times N_{F} \rightarrow F+N_{F}$. So we only have to show $\mu\left(F+N_{F}\right) \subset F \times N_{F}$.

Let $f \in F, \nu \in N_{F}$. Then $\nu \in N_{C}(f)$ by Proposition 6.1. So $f+\nu \in$ $\left(I+N_{C}\right)(f)$ hence, by [1, Ex. 2.8.2], $f=\pi_{C}(f+\nu)$. So $\mu(f+\nu)=$ $(f, \nu) \in F \times N_{F}$.

2. Suppose $F, G$ are nonempty faces of $C$ such that $F+N_{F}=G+N_{G}$. Let $f \in \operatorname{ri} F$ and $\nu \in N_{F}$. Since $f+\nu \in F+N_{F}=G+N_{G}$, we have from part 1 that $f=\pi_{C}(f+\nu) \in G$. So (ri $\left.F\right) \cap G$ is nonempty and it follows, by [17, Thm. 18.1], that $F \subset G$. By symmetry, $G \subset F$, i.e. $F=G$.

We now consider linear subspaces that are either contained in $\operatorname{lin} C$ or orthogonal to par $C$, and show how they can be factored out of $\mathcal{N}_{C}$.

Proposition 9 Let $L, M$ be linear subspaces of $\operatorname{lin} C,(\operatorname{par} C)^{\perp}$ respectively.

1. The mapping $F \mapsto F \cap L^{\perp}$ is a bijection from the faces of $C$ to the faces of $C \cap L^{\perp}$. 
2. Let $c_{0} \in C \cap L^{\perp}$ and $m$ be the dimension of $(L+M)^{\perp}$. The mapping $\sigma \mapsto\left(\sigma-c_{0}\right) \cap(L+M)^{\perp}$ is a bijection from the $n$-cells $\sigma$ of $\mathcal{N}_{C}$ to the $m$-cells of the normal manifold of $\left(C-c_{0}\right) \cap L^{\perp}$ in $(L+M)^{\perp}$.

\section{Proof}

1. Note that, since $L$ is parallel to an affine subspace of $C$,

$$
C=\left(C \cap L^{\perp}\right)+L
$$

in particular $\left(C \cap L^{\perp}\right) \neq \emptyset$. Let $F$ be a face of $C$. If $F \cap L^{\perp}$ is nonempty, let $c_{1}$ and $c_{2}$ belong to $C \cap L^{\perp}$, and $t \in(0,1)$ be such that $t c_{1}+(1-t) c_{2} \in F \cap L^{\perp}$. Then $c_{1}, c_{2} \in F \cap L^{\perp}$ because $F$ is a face and $L^{\perp}$ is convex, that is $F \cap L^{\perp}$ is a face of $C \cap L^{\perp}$. So the mapping $F \mapsto F \cap L^{\perp}$ from the faces $F$ of $C$ to the faces of $C \cap L^{\perp}$ is well defined. For injectivity, suppose $F$ and $F^{\prime}$ are faces of $C$ such that $F \cap L^{\perp}=$ $F^{\prime} \cap L^{\perp}$. Without loss of generality assume that $F$ and $F^{\prime}$ are nonempty. Now $C=\left(C \cap L^{\perp}\right)+L$ hence for any $f \in F \subset C, f+L \subset C$. Let $l \in L$, then $(1 / 2)(f-l)+(1 / 2)(f+l) \in F$, so $f+l \in F$. Hence $f+L \subset F$, i.e. $L$ is parallel to an affine subspace of $F$, and $F=\left(F \cap L^{\perp}\right)+L$. Likewise $L$ is parallel to an affine subspace of $F^{\prime}$, hence

$$
F^{\prime}=\left(F^{\prime} \cap L^{\perp}\right)+L=\left(F \cap L^{\perp}\right)+L=F .
$$

For surjectivity, let $G$ be a nonempty face of $C \cap L^{\perp}$. Since $G \subset L^{\perp}$ we have $(G+L) \cap L^{\perp}=G$. It is left to show that $G+L$ is a face of $C$. 
Now $G+L \subset C$ because $G \subset C \cap L^{\perp}$ and $\left(C \cap L^{\perp}\right)+L=C$. Suppose $c_{1}, c_{2} \in C, t \in(0,1)$ and $c \stackrel{\text { def }}{=} t c_{1}+(1-t) c_{2} \in G+L$. For $i=1,2$ we can decompose $c_{i}$ as the sum $l_{i}+z_{i}$ where $l_{i} \in L$ and $z_{i} \in C \cap L^{\perp}$. As $c \in G+L$ and $G \subset L^{\perp}$, we must have $t z_{1}+(1-t) z_{2} \in G$. Thus $z_{1}, z_{2} \in G, G$ being a face of $C \cap L^{\perp}$, and $c_{1}, c_{2} \in G+L$.

2. We use Proposition 8.2 and part 1 above. Recall that $c_{0}$ belongs to $C \cap L^{\perp}$, and let $C_{0} \stackrel{\text { def }}{=} C-c_{0}$. Note that part 1 applies when $C$ is replaced by $C_{0}$, and we have

$$
\begin{aligned}
C_{0} \cap L^{\perp} & \subset \operatorname{par} C \cap L^{\perp} \\
& \subset M^{\perp} \cap L^{\perp}=(L+M)^{\perp} .
\end{aligned}
$$

Now consider the following mapping from $\mathcal{N}_{C}$ to the normal manifold of $C_{0} \cap L^{\perp}$ in $(L+M)^{\perp}$ : given $\sigma \in \mathcal{N}_{C}$, take the unique face $F$ of $C$ such that $\sigma=F+N_{F}$, map it to the face $G \stackrel{\text { def }}{=} F \cap L^{\perp}$ of $C \cap L^{\perp}$, then map $G$ to the face $G_{0} \stackrel{\text { def }}{=} G-c_{0}$ of $\left(F-c_{0}\right) \cap L^{\perp}$, and finally map $G_{0}$ to the sum of $G_{0}$ and the normal cone, in $(L+M)^{\perp}$, of $C_{0} \cap L^{\perp}$ on $G_{0}$. This mapping is well defined and, as the composition of bijective mappings, is itself bijective. Therefore, any surjective mapping from (the finite set) $\mathcal{N}_{C}$ to the normal manifold of $C_{0} \cap L^{\perp}$ in $(L+M)^{\perp}$ must be bijective.

Let $F$ be a face of $C$, so $F_{0} \stackrel{\text { def }}{=} F-c_{0}$ is a face of $C_{0}$ and $F_{0} \cap L^{\perp}$ is a face of $C_{0} \cap L^{\perp}$. Since $F=\left(F \cap L^{\perp}\right)+L,[16$, Thm. 6.7] yields

$$
\text { ri } F=\operatorname{ri}\left(F \cap L^{\perp}\right)+\operatorname{ri} L=\operatorname{ri}\left(F \cap L^{\perp}\right)+L,
$$


and, in particular, ri $F$ contains $\operatorname{ri}\left(F \cap L^{\perp}\right)$. Choose $g_{0} \in \operatorname{ri}\left(F_{0} \cap L^{\perp}\right)$, so $g_{0}+c_{0} \in \operatorname{ri}\left(F \cap L^{\perp}\right)$ and, by Proposition 6.1,

$$
N_{F}=N_{C}\left(g_{0}+c_{0}\right)=N_{C \cap L^{\perp}}\left(g_{0}+c_{0}\right) \cap L^{\perp}=N_{C_{0} \cap L^{\perp}}\left(g_{0}\right) \cap L^{\perp},
$$

where the second equality follows from the identity $C=\left(C \cap L^{\perp}\right)+L$. Also $F_{0}=\left(F_{0} \cap L^{\perp}\right)+L$, so we have

$$
\begin{aligned}
{[F} & \left.+N_{F}-c_{0}\right] \cap(L+M)^{\perp} \\
& =\left[F_{0} \cap L^{\perp}+L+N_{C_{0} \cap L^{\perp}}\left(g_{0}\right) \cap L^{\perp}\right] \cap\left(L^{\perp} \cap M^{\perp}\right) \\
& =\left[F_{0} \cap L^{\perp}+N_{C_{0} \cap L^{\perp}}\left(g_{0}\right) \cap L^{\perp}\right] \cap M^{\perp}, \\
& =F_{0} \cap L^{\perp}+\left[N_{C_{0} \cap L^{\perp}}\left(g_{0}\right) \cap L^{\perp} \cap M^{\perp}\right], \text { since } F_{0} \cap L^{\perp} \subset M^{\perp}, \\
& =F_{0} \cap L^{\perp}+\left[N_{C_{0} \cap L^{\perp}}\left(g_{0}\right) \cap(L+M)^{\perp}\right] .
\end{aligned}
$$

We already know that $F_{0} \cap L^{\perp}$ is a face of $C_{0} \cap L^{\perp}$. Moreover, by choice of the $g_{0}, N_{C_{0} \cap L^{\perp}}\left(g_{0}\right) \cap(L+M)^{\perp}$ is the normal cone, in $(L+M)^{\perp}$, of $C_{0} \cap L^{\perp}$ on $F_{0} \cap L^{\perp}$.

We have shown that $\sigma \mapsto\left(\sigma-c_{0}\right) \cap(L+M)^{\perp}$ defines a mapping from the $n$-cells $\sigma$ of $\mathcal{N}_{\mathcal{C}}$ to the $m$-cells of the normal manifold of $C_{0} \cap L^{\perp}$ in $(L+M)^{\perp}$; and that, given part 1 , this mapping is surjective. The proof is complete.

When $0 \in C$, i.e. lin $C$ is the largest subspace contained in $C$, part 2 of the last proposition suggests the idea of a quotient of a normal manifold. 
Definition 10 Let $L, M$ be linear subspaces of $C,(\operatorname{par} C)^{\perp}$ respectively. The quotient of $\mathcal{N}_{C}$ over $L+M$, denoted $\mathcal{N}_{C} /(L+M)$, is the normal manifold of $C \cap L^{\perp}$ in $(L+M)^{\perp}$.

This can easily be extended to the situation in which $L$ is a subspace of $\operatorname{lin} C$ but not of $C$. We will not need such generality for our investigation of branching numbers, however.

Next we examine the local structure of $\mathcal{N}_{C}$.

Proposition 11 Let $\hat{x} \in \mathbb{R}^{n}$, and $K$ be the critical cone to $C$ at $\hat{x}$.

1. The mapping $F \mapsto T_{F}\left(\pi_{C}(\hat{x})\right)$ is a bijection from the faces $F$ of $C$ such that $\hat{x} \in F+N_{F}$, to the nonempty faces of $K$.

2. The mapping $\sigma \mapsto T_{\sigma}(\hat{x})$ is bijection from the $n$-cells of $\mathcal{N}_{C}$ that contain $\hat{x}$ to the $n$-cells of $\mathcal{N}_{K}$.

\section{Proof}

1. Let $\hat{c} \stackrel{\text { def }}{=} \pi_{C}(\hat{x})$ and $\hat{\nu} \stackrel{\text { def }}{=} \hat{x}-\pi_{C}(\hat{x})$. Let $\mathcal{F}(\hat{x})$ be the family of faces $F$ of $C$ such that $\hat{x} \in F+N_{F}$. Observe that for each face $F$ of $C$ we have $F=($ aff $F) \cap C: F \subset($ aff $F) \cap C$, and for any $x \in($ aff $F) \cap C$, take $f \in \operatorname{ri} F$ and observe for sufficiently small $t>0$ that $(1-t) f+t x \in F$, hence $x \in F$. Note that each $F \in \mathcal{F}(\hat{x})$ is polyhedral convex because $C$ is polyhedral convex. We will also use the fact that for a polyhedral convex set $D$ and each $d \in D, T_{D}(d)=\operatorname{cone}(D-d)$, where for any nonempty set $S \subset \mathbb{R}^{n}$, cone $(S) \stackrel{\text { def }}{=}\{\alpha s \mid \alpha>0, s \in S\}$. 
Let $F \in \mathcal{F}(\hat{x})$; by Proposition 8.1, $\hat{c} \in F$ and $\hat{\nu} \in N_{F}$. We will show that $G \stackrel{\text { def }}{=} T_{F}(\hat{c})$ is a face of $K$. By Proposition 6.1, $N_{F} \subset(F-\hat{c})^{\perp}$, hence $F-\hat{c} \subset \hat{\nu}^{\perp}$. In particular,

$$
G=\operatorname{cone}(F-\hat{c}) \subset[\operatorname{cone}(C-\hat{c})] \cap \hat{\nu}^{\perp}=K .
$$

Next suppose $k_{1}, k_{2} \in K$ and $t \in(0,1)$ are such that $k \stackrel{\text { def }}{=} t k_{1}+(1-$ $t) k_{2} \in G$. Choose $\alpha>0$ small enough such that

$$
\begin{aligned}
\hat{c}+\alpha k_{1}, \hat{c}+\alpha k_{2} & \in C, \\
\hat{c}+\alpha k & \in F .
\end{aligned}
$$

Since $\hat{c}+\alpha k$ equals $t\left(\hat{c}+\alpha k_{1}\right)+(1-t)\left(\hat{c}+\alpha k_{2}\right)$ and $F$ is a face of $C$, we see that $\alpha k_{1}, \alpha k_{2} \in(F-\hat{c})$. Therefore $k_{1}, k_{2} \in G$, and $G$ is a face of $K$. We have shown that the mapping $F \mapsto T_{F}(\hat{c})$ from $\mathcal{F}(\hat{x})$ to the nonempty faces of $K$ is well defined.

For injectivity, suppose $T_{F}(\hat{c})=T_{F^{\prime}}(\hat{c})$ where $F, F^{\prime} \in \mathcal{F}(\hat{x})$. So for each $f \in F$ there is $\alpha \in(0,1)$ such that $\alpha(f-\hat{c}) \in F^{\prime}-\hat{c}$, hence $(1-\alpha) \hat{c}+\alpha f \in F^{\prime}$. As $F^{\prime}$ is a face of $C, f \in F^{\prime}$, i.e. $F \subset F^{\prime}$. By symmetry, $F \supset F^{\prime}$, and $F=F^{\prime}$.

For surjectivity, let $G$ be a nonempty face of $K$. Since $K$ is a cone, $G$ is a cone too. Let $F \stackrel{\text { def }}{=}(G+\hat{c}) \cap C$. As $G+\hat{c}$ and $C$ are polyhedral convex sets containing $\hat{c}$, we can decompose the tangent cone of $F$ at $\hat{c}$ :

$$
T_{F}(\hat{c})=[\operatorname{cone} G] \cap[\operatorname{cone}(C-\hat{c})]=G \cap T_{C}(\hat{c}) .
$$

Therefore $T_{F}(\hat{c})=G$, so it is only left to be shown that $F \in \mathcal{F}(\hat{x})$. 
First we note that $K=T_{\hat{F}}(\hat{c})$, where $\hat{F} \stackrel{\text { def }}{=} C \cap\left(\hat{c}+\hat{\nu}^{\perp}\right): \hat{c} \in \hat{F}$ and

$$
T_{\hat{F}}(\hat{c})=\operatorname{cone}\left[(C-\hat{c}) \cap \hat{\nu}^{\perp}\right]=[\operatorname{cone}(C-\hat{c})] \cap \hat{\nu}^{\perp}=K .
$$

Second, as $G$ is a face of $T_{\hat{F}}(\hat{c}),(G+\hat{c}) \cap \hat{F}$ is a face of $\hat{F}:$ If $f_{1}, f_{2} \in \hat{F}$ and $t \in(0,1)$ are such that $f \stackrel{\text { def }}{=} t f_{1}+(1-t) f_{2} \in(G+\hat{c}) \cap \hat{F}$, then $f_{1}-\hat{c}, f_{2}-\hat{c} \in T_{\hat{F}}(\hat{c})$, and $t\left(f_{1}-\hat{c}\right)+(1-t)\left(f_{2}-\hat{c}\right)$ equals $f-\hat{c} \in G$. So $f_{1}, f_{2} \in(G+\hat{c}) \cap \hat{F}$.

Third, $(G+\hat{c}) \cap \hat{F}=F:$ since $G \cap(C-\hat{c}) \subset G \subset K \subset \hat{\nu}^{\perp}$, we get

$$
F=(G+\hat{c}) \cap C=(G+\hat{c}) \cap C \cap\left(\hat{c}+\hat{\nu}^{\perp}\right)=(G+\hat{c}) \cap \hat{F} .
$$

Fourth, we show $\hat{F}$ is a face of $C$, for then $F$, which we have shown is a face of $\hat{F}$, must also be a face of $C$. Recall that, by construction, $\hat{\nu} \in N_{C}(\hat{c})$. Let $c_{1}, c_{2} \in C, t \in(0,1), c \stackrel{\text { def }}{=} t c_{1}+(1-t) c_{2} \in \hat{F}$ and assume $c_{1} \notin \hat{F}$. Then $\left\langle c_{1}-\hat{c}, \hat{\nu}\right\rangle<0$ and, as $\langle c-\hat{c}, \hat{\nu}\rangle=0,\left\langle c_{2}-\hat{c}, \hat{\nu}\right\rangle>0$ which contradicts $\hat{\nu} \in N_{C}(\hat{c})$. So $\hat{F}$ is a face of $C$ as needed.

It only remains to be seen that $\hat{x} \in F+N_{F}$. We already know that $\hat{x}=$ $\hat{c}+\hat{\nu}$, where $\hat{c} \in F$ and $F-\hat{c} \subset \hat{F}-\hat{c} \subset \hat{\nu}^{\perp}$. Thus $\hat{\nu} \in(F-\hat{c})^{\perp} \cap N_{C}(\hat{c})$, and Proposition 6.1 assures us that $\hat{\nu} \in N_{F}$.

2. The proof proceeds in a fashion similar to the proof of Proposition 9.2. We will use the proof of part 1 without reference.

Denote by $\mathcal{N}_{C}(\hat{x})$ the family of $n$-cells of $\mathcal{N}_{C}$ that contain $\hat{x}$. Let $\sigma \in$ $\mathcal{N}_{C}(\hat{x})$ and, using Proposition 8.2, $F$ be the unique face of $C$ such that 
$\sigma=F+N_{F}$. Then $\hat{c} \in F$ and $G \stackrel{\text { def }}{=} T_{F}(\hat{c})$ is a face of $K$. The mapping of $\sigma$ to the sum of $G$ and the normal cone to $K$ on $G$ therefore defines a function from $\mathcal{N}_{C}(\hat{x})$ to $\mathcal{N}_{K}$. This function is the composition of invertible mappings, hence is invertible. It follows that any surjective mapping from $\mathcal{N}_{C}(\hat{x})$ to $\mathcal{N}_{K}$ is bijective.

Let $\sigma$ and $F$ be as above, so $\hat{c} \in F$ and $\hat{\nu} \in N_{F}$. Consider

$$
\begin{aligned}
T_{\sigma}(\hat{x}) & =\operatorname{cone}\left[(F-\hat{c})+\left(N_{F}-\hat{\nu}\right)\right] \\
& =\operatorname{cone}(F-\hat{c})+\operatorname{cone}\left(N_{F}-\hat{\nu}\right)
\end{aligned}
$$

since $F-\hat{c}, N_{F}-\hat{\nu}$ are polyhedral convex and contain zero. Also, as $N_{F}$ is a cone containing $\hat{\nu}, \operatorname{cone}\left(N_{F}-\hat{\nu}\right)$ equals $N_{F}+\operatorname{span}(\hat{\nu})$, where $\operatorname{span}(\hat{\nu}) \stackrel{\text { def }}{=}\{\alpha \hat{\nu} \mid \alpha \in \mathbb{R}\}$. So

$$
T_{\sigma}(\hat{x})=T_{F}(\hat{c})+N_{F}+\operatorname{span}(\hat{\nu})
$$

Recall that $T_{F}(\hat{c})$ is face of $K$, and, by definition, $K=T_{C}(\hat{c}) \cap \hat{\nu}^{\perp}$. Let $N$ be the normal cone to $K$ on $T_{F}(\hat{c})$. By Proposition 6.1,

$$
\begin{aligned}
N & =N_{K}(0) \cap\left[T_{F}(\hat{c})-0\right]^{\perp} \\
& =\left[T_{C}(\hat{c}) \cap \hat{\nu}^{\perp}\right]^{\circ} \cap[\operatorname{cone}(F-\hat{c})]^{\perp} \\
& =\left[N_{C}(\hat{c})+\operatorname{span}(\hat{\nu})\right] \cap[F-\hat{c}]^{\perp}
\end{aligned}
$$

since $T_{C}(\hat{c})$ and $\hat{\nu}^{\perp}$ are polyhedral convex cones, and $\left(\hat{\nu}^{\perp}\right)^{\circ}=\operatorname{span}(\hat{\nu})$. Now $\hat{\nu} \in N_{F} \subset(F-\hat{c})^{\perp}$, so $\operatorname{span}(\hat{\nu}) \subset(F-\hat{c})^{\perp}$ and we get

$$
N=\left[N_{C}(\hat{c}) \cap(F-\hat{c})^{\perp}\right]+\operatorname{span}(\hat{\nu})=N_{F}+\operatorname{span}(\hat{\nu}),
$$


where the second equality follows from Proposition 6.1. We deduce that $\sigma \mapsto T_{\sigma}(\hat{x})$ defines a mapping from the $n$-cells $\sigma$ of $\mathcal{N}_{C}(\hat{x})$ to the $n$-cells of $\mathcal{N}_{K}$; and, given part 1 , that this mapping is a surjection. Hence the mapping is bijective.

We have arrived at our main result.

Proposition 12 Suppose $G$ is a nonempty face of $F+N_{F}$ of codimension $m$, where $F$ is a face of $C$. Let $\hat{x} \in \operatorname{ri} G$ and $K$ be the critical cone to $C$ at $\hat{x}$. Define $\hat{c} \stackrel{\text { def }}{=} \pi_{C}(\hat{x}), \hat{\nu} \stackrel{\text { def }}{=} \hat{x}-\hat{c}$,

$$
L \stackrel{\text { def }}{=} \operatorname{par}[(G-\hat{\nu}) \cap F], M \stackrel{\text { def }}{=} \operatorname{par}\left[(G-\hat{c}) \cap N_{F}\right] .
$$

Then $L \subset K, M \subset K^{\perp}, L+M=\operatorname{par} G$, and there is a one-to-one correspondence between the $n$-cells of $\mathcal{N}_{C}$ containing $\hat{x}$ and the $m$-cells of $\mathcal{N}_{K} /(L+M)$.

Proof If $L \subset K, M \subset K^{\perp}$, and $L+M=\operatorname{par} G$, then the dimension of $(L+M)^{\perp}$ is $m$, and the bijective correspondence between the $n$-cells of $\mathcal{N}_{C}$ containing $\hat{x}$ and the $m$-cells of $\mathcal{N}_{K} /(L+M)$ follows from Propositions 11.2 and 9.2.

We will show

$$
G=[(G-\hat{\nu}) \cap F]+\left[(G-\hat{c}) \cap N_{F}\right] .
$$

Let $c+\nu \in G$ where $c \in F$ and $\nu \in N_{F}$, then $c+\hat{\nu}, \hat{c}+\nu \in F+N_{F}$ and, by convexity of $G$,

$$
(1 / 2)(c+\hat{\nu})+(1 / 2)(\hat{c}+\nu)=(1 / 2)(c+\nu)+(1 / 2)(\hat{c}+\hat{\nu}) \in G .
$$


So $c+\hat{\nu}$ and $\hat{c}+\nu$ belong to $G$, and it follows that $c+\nu$ lies in $[(G-\hat{\nu}) \cap$ $F]+\left[(G-\hat{c}) \cap N_{F}\right]$. Conversely if $c \in(G-\hat{\nu}) \cap F$ and $\nu \in(G-\hat{c}) \cap N_{F}$, then both $c+\hat{\nu}$ and $\hat{c}+\nu$ belong to $G$, and an argument similar to the one above yields $c+\nu \in G$.

Recall (Proposition 6.1) that $N_{F}=N_{C}(\hat{c}) \cap(F-\hat{c})^{\perp}$, so par $F$ and $\operatorname{par} N_{F}$ are orthogonal. Hence the decomposition of $\hat{x}$ as the sum $c+\nu$, where $c \in(G-\hat{\nu}) \cap F$ and $\nu \in(G-\hat{c}) \cap N_{F}$, implies $c$ and $v$ are unique, thus $c=\hat{c}$ and $\nu=\hat{\nu}$. Using (1) and [17, Thm. 6.7], we can express ri $G$ is the sum of $\operatorname{ri}[(G-\hat{\nu}) \cap F]$ and $\operatorname{ri}\left[(G-\hat{c}) \cap N_{F}\right]$. As $\hat{x} \in \operatorname{ri} G$ it now follows that $\hat{c}$ and $\hat{\nu}$ are relative interior points of $(G-\hat{\nu}) \cap F$ and $(G-\hat{c}) \cap N_{F}$, respectively.

Therefore we have, in the notation of the previous proof,

$$
L=\operatorname{cone}([(G-\hat{\nu}) \cap F]-\hat{c}),
$$

so $L \subset \operatorname{cone}(C-\hat{c})=T_{C}(\hat{c})$. Also $\hat{\nu}$ belongs to $N_{F}$ (Proposition 8.1) hence to $(F-\hat{c})^{\perp}$, therefore $\hat{\nu}^{\perp} \supset \operatorname{cone}(F-\hat{c}) \supset L$. This shows that the critical cone $K \stackrel{\text { def }}{=} T_{C}(\hat{c}) \cap \hat{\nu}^{\perp}$ contains $L$. Similarly,

$$
M=\operatorname{cone}\left(\left[(G-\hat{c}) \cap N_{F}\right]-\hat{\nu}\right),
$$

so $M \subset \operatorname{cone}\left(N_{F}-\hat{\nu}\right)$. For $\nu \in N_{F} \subset N_{C}(\hat{c})$ and $d \in(C-\hat{c}) \cap \hat{\nu}^{\perp}$, $\langle\nu-\hat{\nu}, d\rangle=\langle\nu, d\rangle \leq 0$, hence

$$
\operatorname{cone}\left(N_{F}-\hat{\nu}\right) \subset\left[\operatorname{cone}(C-\hat{c}) \cap \hat{\nu}^{\perp}\right]^{\circ}=K^{\circ} .
$$

Thus the linear space $M$ lies in $K^{\circ}$, which shows it is a subspace of $K^{\perp}$. 
It only remains to be seen that $\operatorname{par} G=L+M$. This follows from (1), (2), and (3), given that for any nonempty sets $A$ and $B$ in $\mathbb{R}^{n}, \operatorname{par}(A+B)=$ par $A+\operatorname{par} B$. The easy proof of the last fact is left to the reader.

Corollary $13 \mathcal{N}_{C}$ has branching number less than or equal to 4. In particular, $A_{C}$ is pl with respect to a finite pl manifold of $\mathbb{R}^{n}$ that has branching number less than or equal to 4 .

Proof If $n=1$ then, using the convention that the empty set has dimension -1 , the only face of $C$ of codimension 2 is the empty set. Trivially, $\mathcal{N}_{C}$ has cardinality of 3 or less, hence its branching number is less than or equal to 3. (Alternatively, one could ignore the empty set and argue that the idea of branching numbers is vacuous.)

Let $n \geq 2$, and suppose $G$ is a (nonempty) face of $F+N_{F}$ of codimension 2 , where $F$ is a (nonempty) face of $C$. Let $\hat{x}, K, L$ and $M$ be as in Proposition 12. The proposition says that $L+M$ has codimension 2 , and that the number of $n$-cells of $\mathcal{N}_{C}$ containing $G$ (which contains $\hat{x}$ ) is not greater than the number of 2-cells of $\mathcal{N}_{K} /(L+M)$.

Consider $\mathcal{N}_{K} /(L+M)$ which is the normal manifold of a nonempty polyhedral convex cone in a 2-dimensional space. The maximum number of 2-cells of $\mathcal{N}_{K} /(L+M)$ is four: it consists of one 2-cell if $K \cap L^{\perp}$ is $\{0\}$ or $(L+M)^{\perp}$, two if $K \cap L^{\perp}$ is a ray or a halfspace, and four otherwise.

Therefore Robinson's homeomorphism result, Theorem 5, is a corollary of Kuhn and Löwen's homeomorphism result, Theorem 4. 
A minor point is that for a finite $\mathrm{pl}$ manifold $\mathcal{M}$ of $\mathbb{R}$ and a mapping $P: \mathbb{R} \rightarrow \mathbb{R}$ that is $\mathrm{pl}$ with respect to $\mathcal{M}$, it is almost trivial that $P$ is a homeomorphism if and only if it is both proper and coherently oriented. In other words the branching number of $\mathcal{M}$, which equals the cardinality of $\mathcal{M}$ if the empty set is assigned codimension of 2 , has no bearing on whether or not $P$ is a homeomorphism. However, as Kuhn and Löwen [8, p. 122] note, in all higher dimensions the upper bound of 4 on the branching number is tight: there are coherently oriented maps that are $\mathrm{pl}$ with respect to finite $\mathrm{pl}$ manifolds of branching number 5 , but that are not homeomorphic.

\section{Acknowledgement}

I am grateful to Stephen M. Robinson for comments including a simpler proof of Proposition 8.1, and mention of the references [3] and [13].

\section{References}

[1] Brézis, H. (1973). Opérateurs Maximaux Monotones et Semi-groupes de Contractions dans les Espaces de Hilbert. North-Holland Mathematics Studies No. 5, North-Holland, Amsterdam.

[2] Burke, J.V., and Moré, J.J. (1988). On the Identification of Active Constraints. SIAM J. Numer. Anal. 25 1197-1211.

[3] Cottle, R.C., Pang, J.S., and Stone, R.E. (1992). The Linear Complementarity Problem. Academic Press, Boston.

[4] Eaves, B.C. (1976). A Short Course in Solving Equations with PL Homotopies. In: R.W. Cottle and C.E. Lemke, eds., Nonlinear Programming 
(SIAM-AMS Proceedings, v. 9 73-143). American Mathematical Society, Providence, RI.

[5] Eaves, B.C., and Rothblum, U.G. (1990). Relationships of Properties of Piecewise Affine Maps over Ordered Fields. Linear Algebra Appl. 132 1-63.

[6] Fujisawa, T., and Kuh, E.S. (1972). Piecewise-Linear Theory of Resistive Networks. SIAM J. Applied Math. 22 307-328.

[7] Kojima M., and Saigal, R. (1980). On the Relationship between Conditions that Insure a PL Mapping is a Homeomorphism. Math. Operations Res. 5 101-109.

[8] Kuhn, D., and Löwen, R. (1987). Piecewise Affine Bijections of $\mathbb{R}^{n}$, and the Equation $S x^{+}-T x^{-}=y$. Linear Algebra Appl. 96 109-129.

[9] Murty, K.G. (1972). On the Number of Solutions of the Complementarity Problem and Spanning Properties of Cones. Linear Algebra Appl. 5 65-108.

[10] Ralph, D. (1990). A New Proof of Robinson's Homeomorphism Theorem for Piecewise Linear Maps. Ch. 4 in Rank-1 Support Functionals and the Rank-1 Generalized Jacobian, Piecewise Linear Homeomorphisms. Ph.D. Thesis, Computer Sciences Technical Report \#938, Computer Sciences Dept., Univ. of Wisconsin, Madison, WI. To appear in Linear Algebra Appl.

[11] Ralph, D. (1990). Global Convergence of Damped Newton's Method for Nonsmooth Equations, via the Path Search. Technical Report TR 90- 
1181, Dept. of Computer Science, Cornell Univ., Ithaca, NY. To appear in Math. Operations Res.

[12] Rheinboldt, W.C., and Vandergraft, J.S. (1975). On Piecewise Affine Mappings in $\mathbb{R}^{n}$. SIAM J. Applied Math. 29 680-689.

[13] Robinson, S.M. (1987). Local Structure of Feasible Sets in Nonlinear Programming, Part III: Stability and Sensitivity. Math Prog. Study 30 45-66.

[14] Robinson, S.M. (1990). Normal Maps Induced by Linear Transformations. Manuscript, Dept. of Industrial Engineering, Univ. of Wisconsin, Madison, WI. To appear in Math. Operations Res.

[15] Robinson, S.M. (1992). Nonsingularity and Symmetry for Linear Normal Maps. Manuscript, Dept. of Industrial Engineering, Univ. of Wisconsin, Madison, WI.

[16] Robinson, S.M. (1991). An Implicit Function Theorem for a Class of Nonsmooth Functions. Math. Operations Res. 16 292-309.

[17] Rockafellar, R.T. (1970). Convex Analysis. Princeton University Press, Princeton, NJ.

[18] Samelson, H., Thrall, R.M., and Wesler, O. (1958). A Partition Theorem for Euclidean $n$-space. Proc. Amer. Math. Soc. 9 805-807.

[19] Schramm, R. (1980). On Piecewise Linear Functions and Piecewise Linear Equations. Math. Operations Res. 5 510-522. 